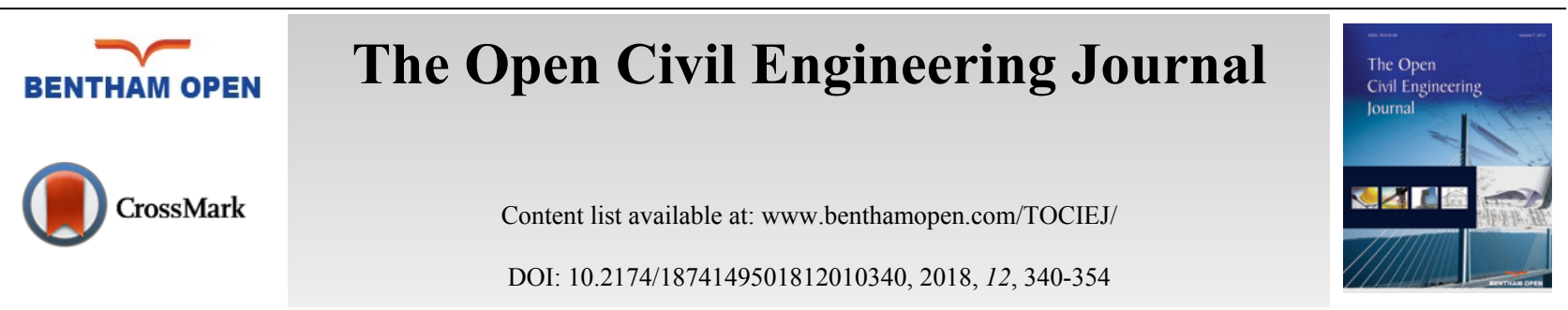

REVIEW ARTICLE

\title{
A Review on the Characteristics of the Smear Zone: Field Data Back Calculation Compared with Laboratory Testing
}

\author{
Rudi Iskandar, A Perwira Mulia Tarigan ${ }^{*}$ and Roesyanto \\ Department of Civil Engineering, Post Graduate Study Program, Faculty of Engineering, University of Sumatera \\ Utara, Kampus USU, Medan 20155, Indonesia
}

Received: September 3, 2018

Revised: October 2, 2018

Accepted: October 18, 2018

\begin{abstract}
:
Background:

The so-called smear zone is a disturbed zone developed in soil during prefabricated vertical drainage (PVD) installation using a mandrel. There are 2 main parameters typically used to characterize the smear zone. First, the extent ratio signifies the extent of the soil damage due to mandrel penetration; and second, the permeability ratio $\kappa$ represents the degree of disturbance to the soil permeability within the smear zone.
\end{abstract}

\section{Objectives:}

This study reviews 2 methods employed by various researchers to obtain the values for the 2 aforementioned parameters, i.e. 1) field data back calculation and 2) laboratory testing. It is found that the $s$ ' values vary between $2.0-5.0$ and between $2.0-6.3$ based on field data back calculation and laboratory testing, consecutively. On the other hand, the $\kappa$ values vary between $2.0-13.8$ and $1.03-$ 3.13 based on field data back calculation and laboratory testing, consecutively.

\section{Results and Conclusion:}

With regard to the previous studies, three causes of the variation are suggested in this study: 1) the laboratory testing's inability to model the complexity of field conditions, 2) the absence of standardized laboratory methods that can be used as references and 3) the level of disturbance in the field which is considerably greater than that in the laboratory and differs at each research location. In addition to the review, historical data of a trial embankment at the embankment of the multi-purpose container yard in Kuala Tanjung, North Sumatra, Indonesia, are exhibited to illustrate the estimation of the $s$ ' and $\kappa$ values.

Keywords: Smear zone, The extent ratio, The permeability ratio, Laboratory testing, Back calculation, Numerical method, PVD installation, Soft clay, Consolidation.

\section{INTRODUCTION}

In the last two decades, the use of a prefabricated vertical drainage known as PVD has been recognized as a very effective and efficient soil improvement method in preloading sites composed of soft soil deposits [1,2]. Smear zone is a disturbed zone in the ground developed during PVD installation using a mandrel. The insertion of the mandrel disturbs the soil around the PVD area to a certain extent and consequently reduces the permeability of the soil $k$ in this area. The permeability reduction in turn inhibits significantly the horizontal consolidation of the soft soil, known as the smear effects which eventually degrade the effectiveness of the PVD [3, 4]. Due to their significant impact, many researchers have been interested in examining the smear zone characteristics [5 - 11].

There are two propositions to delineate the smear zone, i.e. the two-zone and the three-zone hypotheses as presented

* Address correspondence to this author at the Department of Civil Engineering, Post Graduate Study Program, Faculty of Engineering, University of Sumatera Utara, Kampus, Medan 20155, Indonesia; Tel: +628116069333; E-mails: a.perwira@usu.ac.id, a.perwira.mulia@gmail.com 
in Fig. (1). The two-zone hypothesis, described among others by Bergado et al., (1991) [6] and Chai and Miura (1999) [12], divides the ground around the PVD into the smear zone and the undisturbed zone. The three-zone hypothesis, proposed among others by Sharma and Xiao (2000) [3], divides the ground around the PVD into 3 zones, i.e. the smear zone, the transition zone, and the undisturbed zone.

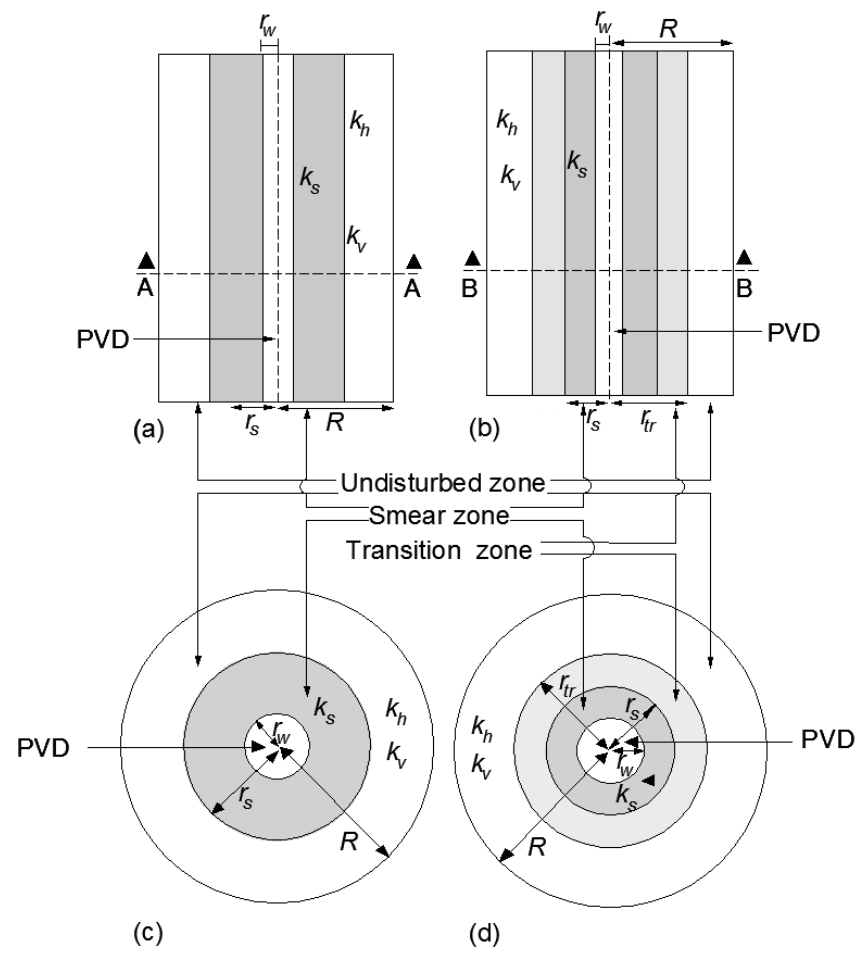

Fig. (1). Schematic figures of a 2-zone theory (a), a 3-zone theory (b) a 2-zone cross section A-A (c), and a 3-zone cross section B-B (d)

The main parameters proposed for characterizing the smear zone are the extent ratio and the permeability ratio. The extent ratio $s$ or $s$ 'is the ratio of the radius of the smear zone $r_{s}$ to the radius of the drain $r_{w}$ or the radius of mandrel $r_{m}$, respectively. Hence $s=r_{s} / r_{w}$ or $s^{\prime}=r_{s} / r_{m}$ represents the range of disturbance due to the penetration of the mandrel on the treated ground. The permeability ratio $\kappa$ is the ratio of the horizontal permeability $k_{h}$ at the undisturbed location to that at the disturbed site $k_{s}$. Hence $\kappa=k_{h} / k_{s}$ represents the degree of disturbance due to penetration of the mandrel on the soil permeability. These ratios become the 2 key values in PVD planning which will in turn affect the rate and duration of consolidation. The values can be estimated using analytical technique, field data back calculation (or numerical method), laboratory testing, or field testing.

A comprehensive standard method for determining them has not yet been established. Field data back calculation (or numerical method) and laboratory testing are among the more frequent applied methods. Researchers who characterized the smear zone with back-calculation of field data did simple curve fitting in their analysis [6 - 10]. Several other researches exercised field data back calculation coupled with finite element analysis [11 - 19], however, field investigation combined with laboratory test could also be conducted for the same purpose [20]. Alternatively investigators performed laboratory testing to obtain the characteristic values [3, 6, 9, 21 - 34]. Those whose results are compared in this study can be seen in column 2 in Tables $\mathbf{1}$ and $\mathbf{2}$, for field data and laboratory testings consecutively.

Due to the limitation of the soil lab model, the $s$ ' and $\kappa$ values resulting from laboratory testing results can not be directly used for planning without proper insights. This study aims to document and evaluate what components in the field that are extremely difficult to model in the laboratory and what disturbances may occur on the ground when the PVD works. This study includes historical data of a trial embankment for an illustrative purpose. It is hoped that the review made in this study will illuminate the subject on the smear zone especially in terms of field data and laboratory testing so that further research and advances in PVD technology may be assisted. 


\section{BASIC THEORY}

\subsection{Analytical Solution}

The settlement for one-dimensional consolidation $S_{p}$ can be given by Eq. (1)

$$
S_{p}=\frac{C_{c} H}{1+e_{o}} \log \left(\frac{P_{o}+\Delta p}{P_{0}}\right)
$$

where $C_{c}=$ compression index, $H=$ layer thickness, $e_{0}=$ initial void ratio, $P_{o}=$ effective vertical stress and $\Delta p=$ additional effective vertical stress. Assuming equal strain and pure radial flowing, the average degree of consolidation on cell units $\bar{U}$, including the smear effect and excluding the well resistance effect, can be expressed analytically as follows [35], (Eq. 2):

$$
\bar{U}=1-\exp \left[\frac{-8 T_{h}}{m}\right]
$$

where $T_{h}=$ the time factor in the horizontal direction and $m=$ the $s$ and $\kappa$ dependent parameters, both of which are expressed as Eq. (3 and 4):

$$
\begin{gathered}
T_{h}=c_{h} t / d_{e}^{2} \\
m=\ln (n / s)-0,75+\left(\frac{k_{h}}{k_{s}}\right) \ln (s)
\end{gathered}
$$

where $n=r_{e} / r_{w}$ and $c_{h}=$ the horizontal consolidation coefficient, and $\mathrm{t}=$ the time in days.

Note that [35] assumed a constant smear effect resulting in constant reduced permeability in the smear zone. Walker and Indraratna (2006) [36] suggested a parabolic distributed smear effect in the smear zone, and they subsequently proposed the expression for $m$ as Eq. (5):

$$
\begin{gathered}
m=\ln (n / s)-0,75+\frac{\kappa(s-1)^{2}}{\left(s^{2}-2 \kappa s+\kappa\right)} \ln \left(\frac{s}{\sqrt{\kappa}}\right) \\
-\frac{\mathrm{s}(\mathrm{s}-1) \sqrt{\kappa(\kappa-1)}}{2\left(\mathrm{~s}^{2}-2 \kappa \mathrm{s}+\kappa\right)} \ln \left(\frac{\sqrt{\kappa+} \sqrt{\kappa-1}}{\sqrt{\kappa})-\sqrt{\kappa-1}}\right)
\end{gathered}
$$

In contrast Rujikiatkamjorn and Indraratna (2009) [37] assumed a linearly distributed smear effect on the smear zone and offered the expression for $m$ as Eq. (6):

$$
m=\ln (n / s)-0,75+\frac{\kappa(s-1)}{(s-\kappa)} \ln \left(-\frac{s}{\kappa}\right)
$$

\subsection{Coefficient of Permeability}

The coefficient of permeability of the soil layer measured in situ using field apparatus is typically greater than that measured in the laboratory using for example oedometer. In this regard, Tavenas et al., (1986) [38] stated the relationship of the permeability coefficients (Eq. 7):

$$
\left(k_{h}\right)_{f}=\left(k_{h}\right)_{l} C_{f} \text { or }\left(k_{v}\right)_{f}=\left(k_{v}\right)_{l} C_{f}
$$


where $k_{h}$ and $k_{v}$ are the horizontal and vertical permeability coefficients, consecutively, subcripts $f$ and $l$ denote values determined in the field and in the laboratory, consecutively, and $C_{f}$ is the ratio of the permeability coefficient from the field to that from the laboratory. The ratio $C_{f}$ depends on the sensitivity of the soil, macropore, and stratification of the soil layer.

As indicated by several researchers, laboratory testing may be the correct way to determine the magnitude of the coefficient of permeability in the smear zone $k_{s}$ Chai and Miura (1999) [12]. However it should be noted that because of the disruption in the sample and the comparatively small sample sizes, the laboratory permeability coefficient $k_{l}$ is less than field permeability coefficient value $k_{f}$. Chai and Miura (1999) [12] admitted many uncertainties that exist in determining the $k_{h} / k_{s}$ ratio in the field and proposed:

$$
\left(k_{h} / k_{s}\right)_{f}=\left(k_{h} / k_{s}\right)_{l} C_{f}
$$

For the planning and the implementation of PVD, the value for $C_{f}$ in Eq.(8) should take into account the disturbance components that affect the permeability in the ground during the PVD work in the field.

\subsection{Geotechnical Instruments}

In order to observe the underground conditions during ground treatment, several geotechnical instruments are installed to measure realistic conditions beneath the soil surface. The commonly installed instruments are pneumatic piezometer for measuring excess pore water pressure, water standpipes for measuring water level, settlement plate for observing surface settlement, extensometer for gauging settlement at certain depths, and inclinometer for perceiving horizontal deformation.

\section{IMPORTANT NOTES OF THE FIELD WORKS}

The field work starts usually from land clearing and stripping. These are followed by spreading the geotextile separator layer and the sand blanket layer on top of the separator layer, and then the sand blanket can be compacted. Subsequently, installation of geotechnical instruments, including pneumatometer piezometer, water standpipe, settlement plate, and inclinometer, is made. Lastly, PVD installation and preloading can be conducted.

The factors that may cause different disturbances to the soil during the PVD work include depth of installation, thickness of sand mat, patterns of installation, PVD interval, mandrel shape and dimension, height of embankment, and speed of installation. These factors vary between locations which are indicated in columns 3 up to 10 in Table $\mathbf{1}$, and their influences on the smear zone characteristics are discussed later in this paper.

Table 1. 16 sets of summary results of ground improvement studies using PVD.

\begin{tabular}{|c|c|c|c|c|c|c|c|c|c|c|c|c|c|}
\hline \multirow[b]{2}{*}{ No. } & \multirow[b]{2}{*}{ Researchers } & \multirow{2}{*}{\begin{tabular}{|c|}
$\begin{array}{c}\text { Depth of } \\
\text { Installation } \\
(\mathrm{m})\end{array}$ \\
\end{tabular}} & \multirow{2}{*}{$\mid \begin{array}{c}\text { Sand Mat } \\
\text { Thickness } \\
(\mathrm{mm})\end{array}$} & \multirow{2}{*}{$\begin{array}{c}\text { Patterns } \\
\text { Installation }\end{array}$} & \multirow{2}{*}{\begin{tabular}{|} 
Installation \\
Distance \\
(m)
\end{tabular}} & \multicolumn{2}{|c|}{ Mandrel } & \multirow{2}{*}{$\begin{array}{c}\text { Height of } \\
\text { Embankment } \\
\text { (m) }\end{array}$} & \multirow{2}{*}{$\begin{array}{c}\text { Speed of } \\
\text { Installation } \\
(\mathrm{mm} / \mathrm{s})\end{array}$} & \multirow[b]{2}{*}{$\begin{array}{l}\text { Type of } \\
\text { Soil }\end{array}$} & \multirow{2}{*}{$\begin{array}{c}\text { Field Data Back- } \\
\text { Calculation }\end{array}$} & \multirow{2}{*}{\begin{tabular}{|c|}
$s^{\prime}=r_{s} / r_{m}$ \\
$o r$ \\
$s=$ \\
$r_{s} / r_{w} *$
\end{tabular}} & \multirow[b]{2}{*}{$\boldsymbol{\kappa}=\boldsymbol{k}_{h} / \boldsymbol{k}_{\mathrm{s}}$} \\
\hline & & & & & & Shape & \begin{tabular}{|c} 
Dimension \\
$\left(\mathrm{mm}^{2}\right)$
\end{tabular} & & & & & & \\
\hline 1 & 2 & 3 & 4 & 5 & 6 & 7 & 8 & 9 & 10 & 11 & 12 & 13 & 14 \\
\hline 1 & $\begin{array}{l}\text { Bergado et al. } \\
\text { (1991) [6] }\end{array}$ & 8.0 & 1,000 & Square & 1.2 & Rectangular & $\begin{array}{l}45 \times 150 \\
150 \times 150\end{array}$ & & & \begin{tabular}{|c|} 
Soft Clay, \\
Bangkok \\
\end{tabular} & Analytical & 2.0 & $k_{h}=k_{v}$ \\
\hline 2 & $\begin{array}{c}\text { Bergado et al. } \\
\text { (1992) [7] }\end{array}$ & 8.0 & 300 & Triangle & 1.5 & Rectangular & $150 \times 45$ & 4.00 & - & \begin{tabular}{|c|} 
Soft Clay, \\
Bangkok \\
\end{tabular} & Analytical & $2.5 *$ & 10.0 \\
\hline 3 & $\begin{array}{l}\text { Bergado et al. } \\
\text { (2000) [8] }\end{array}$ & 12.0 & 1,500 & Square & $1.0 ; 1.2 ; 1.5$ & Rectangular & - & 4.20 & - & \begin{tabular}{|c|} 
Soft Clay, \\
Bangkok \\
\end{tabular} & Analytical & 2.0 & 5.0 \\
\hline 4 & $\begin{array}{c}\text { Saowapakpiboonet } \\
\text { et al. (2010) [9] }\end{array}$ & 10.0 & - & Triangle & 0.85 & Rectangular & - & - & - & \begin{tabular}{|c|} 
Soft Clay, \\
Bangkok \\
Soft Clay, \\
Bangkok \\
\end{tabular} & $\begin{array}{l}\text { Analytical } \\
\text { Analytical }\end{array}$ & $\begin{array}{l}2.0 \\
2.0\end{array}$ & $\begin{array}{c}7.2 \\
6.6 \\
(+v)\end{array}$ \\
\hline 5 & $\begin{array}{l}\text { Hiep and Chung } \\
\text { (2018) [10] }\end{array}$ & $30.0-35.0$ & $500-800$ & Triangle & 1.5 & Rectangular & $120 \times 80$ & $6.00-8.00$ & - & $\begin{array}{c}\text { Clay, } \\
\text { Mekong } \\
\text { Delta }\end{array}$ & Analytical & 2.0 & 2.0 \\
\hline 6 & $\begin{array}{c}\text { Indraratna and } \\
\text { Redana (2000) } \\
{[11]}\end{array}$ & $\begin{array}{l}12.0 \\
18.0\end{array}$ & $\begin{array}{c}1,500 \\
-\end{array}$ & $\begin{array}{l}\text { Square } \\
\text { Triangle }\end{array}$ & $\begin{array}{c}1 ; 1.2 ; 1.5 \\
1.3\end{array}$ & $\begin{array}{l}\text { Rectangular } \\
\text { Rectangular }\end{array}$ & $\begin{array}{l}- \\
-\end{array}$ & $\begin{array}{c}4.20 \\
-\end{array}$ & $\begin{array}{l}- \\
-\end{array}$ & $\begin{array}{c}\text { Soft Clay, } \\
\text { Bangkok } \\
\text { Muar Clay } \\
\text { Deposit } \\
\end{array}$ & $\begin{array}{l}\text { FEM } \\
\text { FEM }\end{array}$ & $\begin{array}{l}4-5 * \\
4-5 *\end{array}$ & $\begin{array}{l}k_{h}=k_{v} \\
k_{h}=k_{v}\end{array}$ \\
\hline 7 & $\begin{array}{c}\text { Chai and Miura } \\
\text { (1999) [12] }\end{array}$ & 25.0 & 500 & Square & 1.5 & Rectangular & - & 3.50 & - & \begin{tabular}{|c|}
$\begin{array}{c}\text { Soft Clay, } \\
\text { Ariake }\end{array}$ \\
\end{tabular} & FEM & 3.0 & 10.0 \\
\hline 8 & $\begin{array}{c}\text { Chai et al. (1995) } \\
{[13]}\end{array}$ & 18.0 & - & Triangle & 1.2 & Rectangular & - & 4.70 & - & $\begin{array}{c}\text { Muar Clay } \\
\text { Deposit }\end{array}$ & FEM & $4.2 *$ & 2.0 \\
\hline
\end{tabular}




\begin{tabular}{|c|c|c|c|c|c|c|c|c|c|c|c|c|c|}
\hline \multirow[b]{2}{*}{ No. } & \multirow[b]{2}{*}{ Researchers } & \multirow{2}{*}{$\begin{array}{c}\text { Depth of } \\
\text { Installation } \\
\text { (m) }\end{array}$} & \multirow{2}{*}{$\begin{array}{c}\text { Sand Mat } \\
\text { Thickness } \\
(\mathrm{mm})\end{array}$} & \multirow{2}{*}{$\begin{array}{c}\text { Patterns } \\
\text { Installation }\end{array}$} & \multirow{2}{*}{$\begin{array}{c}\text { Installation } \\
\text { Distance } \\
\text { (m) }\end{array}$} & \multicolumn{2}{|c|}{ Mandrel } & \multirow{2}{*}{$\begin{array}{c}\text { Height of } \\
\text { Embankment } \\
\text { (m) }\end{array}$} & \multirow{2}{*}{$\begin{array}{c}\text { Speed of } \\
\text { Installation } \\
(\mathrm{mm} / \mathrm{s})\end{array}$} & \multirow{2}{*}{$\begin{array}{l}\text { Type of } \\
\text { Soil }\end{array}$} & \multirow{2}{*}{$\begin{array}{c}\text { Field Data Back- } \\
\text { Calculation }\end{array}$} & \multirow{2}{*}{$\begin{array}{c}s^{\prime}=r_{s} / r_{m} \\
o r \\
s= \\
r_{s} / r_{w} *\end{array}$} & \multirow[b]{2}{*}{$\boldsymbol{\kappa}=\boldsymbol{k}_{l} / \boldsymbol{k}_{s}$} \\
\hline & & & & & & Shape & $\begin{array}{c}\text { Dimension } \\
\left(\mathrm{mm}^{2}\right)\end{array}$ & & & & & & \\
\hline 9 & $\begin{array}{c}\text { Chai et al. (1996) } \\
{[14]}\end{array}$ & $\begin{array}{l}18.0 \\
12.0\end{array}$ & - & $\begin{array}{l}\text { Triangle } \\
\text { Square }\end{array}$ & $\begin{array}{l}1.2 \\
1.5\end{array}$ & $\begin{array}{l}\text { Rectangular } \\
\text { Rectangular }\end{array}$ & $\begin{array}{c}- \\
120 \times 60\end{array}$ & $\begin{array}{c}4.70 \\
-\end{array}$ & $\begin{array}{l}- \\
-\end{array}$ & \begin{tabular}{|c|} 
Muar Clay \\
Deposit \\
Soft Clay, \\
Bangkok
\end{tabular} & $\begin{array}{l}\text { FEM } \\
\text { FEM }\end{array}$ & $\begin{array}{l}3.0 \\
3.0\end{array}$ & $\begin{array}{l}10.0 \\
10.0\end{array}$ \\
\hline 10 & $\begin{array}{c}\text { Chai et al. (2001) } \\
{[15]}\end{array}$ & 19.0 & 500 & Triangle & 1.5 & Rectangular & - & - & - & $\begin{array}{l}\text { Mucky } \\
\text { Clay }\end{array}$ & FEM & $6.7 *$ & 13.8 \\
\hline 11 & $\begin{array}{c}\text { Indraratna et al. } \\
(2008)[16]\end{array}$ & 12.0 & - & Triangle & 1.5 & Rectangular & - & 1.50 & - & $\begin{array}{l}\text { Soft Clay, } \\
\text { Swedish }\end{array}$ & FEM & $2.0 *$ & 4.0 \\
\hline 12 & $\begin{array}{l}\text { Pajouh et al. } \\
\text { (2013) [17] }\end{array}$ & 9.0 & - & Square & 1.0 & Rectangular & - & 3.00 & - & \begin{tabular}{|l|} 
Soft Clay, \\
Chittagong
\end{tabular} & FEM & 3.0 & 2.0 \\
\hline 13 & $\begin{array}{c}\text { Pajouh } e t \\
\text { al.(2014) [18] }\end{array}$ & $\begin{array}{c}11.0 \\
9.0\end{array}$ & $\begin{array}{c}650 \\
-\end{array}$ & $\begin{array}{l}\text { Triangle } \\
\text { Square }\end{array}$ & $\begin{array}{l}2.0 \\
1.0\end{array}$ & $\begin{array}{l}\text { Rectangular } \\
\text { Rectangular }\end{array}$ & $\begin{array}{l}- \\
-\end{array}$ & $\begin{array}{l}2.85 \\
3.00\end{array}$ & $\begin{array}{l}- \\
-\end{array}$ & \begin{tabular}{|c|} 
Silty Clay \\
Soft Clay, \\
Chittagong
\end{tabular} & $\begin{array}{l}\text { FEM } \\
\text { FEM }\end{array}$ & $\begin{array}{l}3.0 \\
3.0\end{array}$ & $\begin{array}{l}4.0 \\
2.0\end{array}$ \\
\hline 14 & $\begin{array}{c}\text { Pajouh et } \\
\text { al.(2014) [19] }\end{array}$ & $\begin{array}{c}24.0 \\
22.0 \\
11.00\end{array}$ & $\begin{array}{c}1,500 \\
650 \\
650\end{array}$ & $\begin{array}{l}\text { Square } \\
\text { Triangle } \\
\text { Triangle }\end{array}$ & $\begin{array}{c}1.0 \\
1.35 \\
2.0\end{array}$ & $\begin{array}{l}\text { Rectangular } \\
\text { Rectangular } \\
\text { Rectangular }\end{array}$ & $\begin{array}{l}- \\
- \\
-\end{array}$ & $\begin{array}{l}8.50 \\
5.00 \\
2.80\end{array}$ & $\begin{array}{l}- \\
- \\
-\end{array}$ & \begin{tabular}{|c|} 
Soft Clay, \\
Ballina \\
Soft Soil \\
Silty Clay
\end{tabular} & $\begin{array}{l}\text { FEM } \\
\text { FEM } \\
\text { FEM }\end{array}$ & $\begin{array}{l}4.0 \\
5.0 \\
3.0\end{array}$ & $\begin{array}{l}4.0 \\
5.0 \\
4.0\end{array}$ \\
\hline 15 & $\begin{array}{c}\text { Bo et al. (2003) } \\
{[20]}\end{array}$ & 50.0 & & Square & $2.2,5.3$ & $\begin{array}{c}\text { Rectangular } \\
\text { Rhombus }\end{array}$ & $\begin{array}{l}120 \times 60 \\
145 \times 90\end{array}$ & & $\begin{array}{l}300-600 \\
300-600\end{array}$ & \begin{tabular}{|c|} 
Marine \\
Clay, \\
Singapore
\end{tabular} & $\begin{array}{c}\text { Field and } \\
\text { Laboratory Test }\end{array}$ & $4.0-7.0$ & $2.0-10.0$ \\
\hline 16 & The Case study & 17.0 & 600 & Triangle & 1.5 & Rectangular & $120 \times 50$ & 5.20 & $400-500$ & \begin{tabular}{|c|} 
Soft Soil, \\
North \\
Sumatra \\
\end{tabular} & Analytical & 3.0 & 7.0 \\
\hline
\end{tabular}

$(+\mathrm{v})=$ with PVD + Vacuum Preloading.

As previously mentioned, the characteristics of the smear zone are normally estimated by the back calculation method using the data resulting from the geotechnical instrument. The estimation is conducted based on the obtained time dependent settlement data which are then fitted with Eq. 1 or processed with a numerical program. However, Bo et al., (2001) [39] did field testing to determine the values of $s$ ' and $\kappa$ using field apparatus. Table 1 summarizes 16 data set on soil improvement conditions from various locations using PVD. Note in the table that the extent ratio and the permeability ratio are given in the last two columns.

\section{IMPORTANT NOTES OF LABORATORY WORKS}

Tests in the lab are conducted without referential standards. Nevertheless, the shapes of laboratory soil tanks used are typically cylinders and boxes. Previous researchers had different $h / d$ ratios from one and another as shown in column 3 in Table 2 . Note that $h$ and $d$ are the height and the diameter of the cylindrical soil tank, consecutively. In contrast, Tran-Nguyen and Edil (2011) [26] developed the box-shape tank which then further utilized by Sengul, et al. (2016) [33]. Before placed in the soil tank, the sample is tested for the basic soil properties including moisture content $w$, liquid limit $L_{L}$, plastic limit $P_{L}$, void ratio $e$, spesific gravity $G_{s}$, unit weight soil $\gamma$, compression index $c_{c}$, and swelling index $c_{s}$.

Tabel 2. 17 sets of summary results of laboratory testing to determine smear zone characteristics.

\begin{tabular}{|c|c|c|c|c|c|c|c|c|c|c|}
\hline \multirow[b]{2}{*}{ No. } & \multirow[b]{2}{*}{ Researchers } & \multirow[b]{2}{*}{$\begin{array}{c}\text { Tank } \\
\text { Dimension }\end{array}$} & \multirow[b]{2}{*}{$\begin{array}{l}\text { Basic Soil } \\
\text { Properties }\end{array}$} & \multirow{2}{*}{ Sample Preparation } & \multirow{2}{*}{$\begin{array}{l}\text { Stress } \\
(\mathrm{kPa})\end{array}$} & \multirow{2}{*}{$\begin{array}{c}\text { PVD } \\
\text { Dimension }\end{array}$} & \multirow{2}{*}{$\begin{array}{c}\text { Mandrel } \\
\left(\mathrm{mm}^{2}\right)\end{array}$} & \multirow{2}{*}{$\begin{array}{c}\text { Speed of } \\
\text { Installation }\end{array}$} & \multirow{2}{*}{\begin{tabular}{|c|} 
Extent \\
Ratio \\
$\boldsymbol{s}^{\prime}=r_{s} / r_{m}$ \\
$o r$ \\
$s=$ \\
$r_{s} / r_{w} *$ \\
\end{tabular}} & \multirow[b]{2}{*}{$\begin{array}{c}\text { Permeability } \\
\text { Ratio } \\
\boldsymbol{\kappa}=\boldsymbol{k}_{l} / \boldsymbol{k}_{s}\end{array}$} \\
\hline & & & & & & & & & & \\
\hline 1 & 2 & 3 & 4 & 5 & 6 & 7 & 8 & 9 & 10 & 11 \\
\hline 1 & $\begin{array}{c}\text { Sharma and Xiao } \\
(2000)[3] \\
3 \text { zones }\end{array}$ & $\begin{array}{c}h=400 \mathrm{~mm} \\
d=1000 \mathrm{~mm} \\
h / d=0.40\end{array}$ & $\begin{array}{c}\text { Reconstituted } \\
\text { Kaolinite } \\
w=65 \%, L_{L}=70 \%, \\
P_{L}=40 \%, \\
e=1.4, G_{s}=2.61\end{array}$ & $\begin{array}{c}\text { Kaolinite is mixed } \\
\text { water up to } w=2 \mathrm{x} \\
L_{L} \text {, put into } \\
\text { consolidation tank } \\
\text { and be } \\
\text { vacuumed for stress } \\
\text { at } 90 \mathrm{kPa} \text { for several } \\
\text { days. }\end{array}$ & $\begin{array}{c}P_{o}= \\
100 \Delta_{P} \\
= \\
1,102\end{array}$ & $\begin{array}{c}\mathrm{SD} \\
\operatorname{diam} .=50 \\
\mathrm{~mm}\end{array}$ & $\begin{array}{c}\text { Circular } \\
\text { diam. } \\
=50 \mathrm{~mm}\end{array}$ & 5.0 & $4.0 *$ & 1.3 \\
\hline
\end{tabular}




\begin{tabular}{|c|c|c|c|c|c|c|c|c|c|c|}
\hline \multirow[b]{2}{*}{ No. } & \multirow[b]{2}{*}{ Researchers } & \multirow[b]{2}{*}{$\begin{array}{c}\text { Tank } \\
\text { Dimension }\end{array}$} & \multirow[b]{2}{*}{$\begin{array}{l}\text { Basic Soil } \\
\text { Properties }\end{array}$} & \multirow[b]{2}{*}{ Sample Preparation } & \multirow{2}{*}{\begin{tabular}{|l|} 
Stress \\
(kPa)
\end{tabular}} & \multirow{2}{*}{$\begin{array}{c}\text { PVD } \\
\text { Dimension } \\
\\
\left(\mathrm{mm}^{2}\right)\end{array}$} & \multirow{2}{*}{$\begin{array}{c}\text { Mandrel } \\
\left(\mathrm{mm}^{2}\right)\end{array}$} & \multirow{2}{*}{$\begin{array}{c}\text { Speed of } \\
\text { Installation } \\
(\mathrm{mm} / \mathrm{s})\end{array}$} & \multirow{2}{*}{\begin{tabular}{|c|} 
Extent \\
Ratio \\
$s^{\prime}=r_{s} / r_{m}$ \\
$o r$ \\
$s=$ \\
$r_{s} / r_{w} *$ \\
\end{tabular}} & \multirow[b]{2}{*}{$\begin{array}{c}\text { Permeability } \\
\text { Ratio } \\
\boldsymbol{\kappa}=\boldsymbol{k}_{h} / \boldsymbol{k}_{s}\end{array}$} \\
\hline & & & & & & & & & & \\
\hline 2 & $\begin{array}{l}\text { Bergado et al. } \\
\text { (1991) [6] } \\
2 \text { zones }\end{array}$ & $\begin{array}{c}h=920 \mathrm{~mm} \\
d=455 \mathrm{~mm} \\
h / d=2.02\end{array}$ & \begin{tabular}{|c} 
Reconstituted Soft \\
Bangkok Clay \\
$\gamma=14.7 \mathrm{kN} / \mathrm{m}^{3}$, \\
$C_{c}=0.80$, \\
$C_{s}=0.13, e=2.3$
\end{tabular} & $\begin{array}{c}\text { Samples are placed in } \\
\text { cell consolidation } \\
\text { layer by layer. Sand } \\
\text { of } 5 \mathrm{~cm} \text { thick is given } \\
\text { on the surface. }\end{array}$ & $\begin{array}{l}P_{o}= \\
10.2 \\
\Delta_{P}= \\
47.8\end{array}$ & $40 \times 6$ & $\begin{array}{l}60 \times 60 \\
\text { Recta- } \\
\text { ngular }\end{array}$ & - & 2.0 & $1.5-2.0$ \\
\hline 3 & $\begin{array}{c}\text { Saowapakpiboon } \\
\text { et al. }(2010)[9] \\
2 \text { zones }\end{array}$ & $\begin{array}{c}h=500 \mathrm{~mm} \\
d=305 \mathrm{~mm} \\
h / d=1.64\end{array}$ & $\begin{array}{c}\text { Reconstituted Soft } \\
\text { Bangkok Clay } \\
w=113 \%, L_{L}= \\
102 \%, P_{L}=40 \%, \\
G_{s}=2.66, \gamma=14.7 \\
\mathrm{kN} / \mathrm{m}^{3}\end{array}$ & $\begin{array}{c}\text { The sample is taken } \\
\text { into } 3-4 \mathrm{~m} \text { from } \\
\text { the ground surface } \\
\text { and placed in the cell } \\
\text { consolidometer } \\
\text { coating. }\end{array}$ & $\begin{array}{c}- \\
\Delta_{P}= \\
100\end{array}$ & $100 \times 3.5$ & - & - & $\begin{array}{c}2.0 \\
2.0 \\
(+v)\end{array}$ & $\begin{array}{l}2.7 \\
2.5\end{array}$ \\
\hline 4 & $\begin{array}{c}\text { Indraratna and } \\
\text { Redana (1998) } \\
{[21]} \\
2 \text { zones }\end{array}$ & $\begin{array}{c}h=950 \mathrm{~mm} \\
d=450 \mathrm{~mm} \\
h / d=2.11\end{array}$ & $\begin{array}{c}\text { Reconstituted } \\
\text { Alluvial Clay, } \\
\text { Sydnay } w=40 \%, \\
L_{L}=70 \%, P_{L}=30 \%, \\
G_{s}=2.6, \gamma=17.0 \\
\mathrm{kN} / \mathrm{m}\end{array}$ & $\begin{array}{c}\text { Samples are mixed } \\
\text { with water, placed in } \\
\text { a consolidometer cell } \\
\text { and compacted } \\
\text { layer by layer. } \\
\text { Surface is given sand } \\
5 \mathrm{~cm} .\end{array}$ & $\begin{array}{c}P_{o}= \\
20 \\
\Delta_{P}= \\
200\end{array}$ & $\begin{array}{c}\text { SD diam. }= \\
46 \mathrm{~mm}\end{array}$ & $\begin{array}{l}\text { Circular } \\
\text { diam. }= \\
50 \mathrm{~mm}\end{array}$ & - & $\begin{array}{l}4.0- \\
5.0\end{array}$ & $\mathrm{k}_{\mathrm{h}} / \mathrm{k}_{\mathrm{v}}=1.15$ \\
\hline 5 & $\begin{array}{l}\text { Indraratna and } \\
\text { Rujikiatkamjorn } \\
\text { (2004) [22]. } \\
2 \text { zones }\end{array}$ & $\begin{array}{c}h=950 \mathrm{~mm} \\
d=450 \mathrm{~mm} \\
h / d=2.11\end{array}$ & $\begin{array}{c}\text { Reconstituted } \\
\text { Alluvial Clay, } \\
\text { Moruya } w= \\
45 \%, L_{L}=42 \%, \\
P_{L}=17 \%, G_{s}=2.6 \\
\gamma=17.0 \mathrm{kN} / \mathrm{m}^{3}\end{array}$ & \begin{tabular}{|c|} 
Samples are mixed \\
with water up to $w$ \\
slightly larger than $L_{L}$, \\
placed in a \\
coating,compacted \\
using consolidometer \\
cell.
\end{tabular} & $\begin{array}{c}P_{o}=20 \\
\Delta_{P}= \\
30+50\end{array}$ & $100 \times 3$ & $\begin{array}{c}125 \times 25 \\
\text { Recta- } \\
\text { ngular }\end{array}$ & - & $\begin{array}{l}3.0 * \\
3.0 * \\
(+\mathrm{v})\end{array}$ & $\begin{array}{c}\mathrm{k}_{\mathrm{h}} / \mathrm{k}_{\mathrm{v}}= \\
1.17-1.20\end{array}$ \\
\hline 6 & $\begin{array}{c}\text { Sathananthan and } \\
\text { Indraratna }(2006) \\
{[23]} \\
2 \text { zones }\end{array}$ & $\begin{array}{c}h=1040 \mathrm{~mm} \\
d=650 \mathrm{~mm} \\
h / d=1.60\end{array}$ & \begin{tabular}{|c|} 
Reconstituted \\
Alluvial Clay, \\
Moruya $w=45 \%$, \\
$L_{L}=42 \%$, \\
$P_{L}=17 \%, G_{s}=2.6$, \\
$\gamma=17.0 \mathrm{kN} / \mathrm{m}^{3}$
\end{tabular} & \begin{tabular}{|c|} 
Clay is mixed with \\
water, kept on \\
container for several \\
days, placed \\
in coating \\
consolidometer cell \\
(150 mm/Layer), and \\
compacted.
\end{tabular} & $\begin{array}{c}P_{o}= \\
20 \\
\Delta_{P}= \\
200\end{array}$ & $100 \times 3$ & $\begin{array}{c}125 \times 25 \\
\text { Recta- } \\
\text { ngular }\end{array}$ & 8.3 & 2.5 & 1.34 \\
\hline 7 & $\begin{array}{c}\text { Feng and } \\
\text { Yin (2006) [24] } \\
2 \text { zones }\end{array}$ & $\begin{array}{c}h=450 \mathrm{~mm} \\
d=300 \mathrm{~mm} \\
h / d=1.50\end{array}$ & \begin{tabular}{|c|} 
Reconstituted \\
Hongkong Marine \\
Clay, $w=85,6 \%$, \\
$L_{L}=51.1 \%$, \\
$P_{L}=26.1 \%$, \\
$G_{s}=2.58$
\end{tabular} & \begin{tabular}{|} 
Clay is mixed with \\
water and stored in \\
container for several \\
days, placed in \\
coating \\
consolidometer cells \\
$(150 \mathrm{~mm} /$ \\
layer $)$ and compacted.
\end{tabular} & $\begin{array}{c}P_{o}= \\
20 \\
\Delta_{P}= \\
80\end{array}$ & $50 \times 5$ & $\begin{array}{l}60 \times 13 \\
\text { Recta- } \\
\text { ngular }\end{array}$ & - & 2.0 & 2.0 \\
\hline 8 & $\begin{array}{c}\text { Shin et al. }(2009) \\
{[25]} \\
2 \text { zones }\end{array}$ & $\begin{array}{c}h=1000 \mathrm{~mm} \\
d=700 \mathrm{~mm} \\
h / d=1.43\end{array}$ & $\begin{array}{c}\text { Reconstituted } \\
\text { Busan Clay } \\
w=56 \%, L_{L}=46.4 \\
\% \\
P_{L}=24.1 \%, G_{s}= \\
2.64\end{array}$ & \begin{tabular}{|c|} 
The test sample \\
passed the sieve \\
No.40. \\
is mixed with water \\
up to $w=2 \times L_{L}$. \\
The trapped air is \\
removed by vacuum \\
during mixing.
\end{tabular} & $\begin{array}{c}P_{o}= \\
50 \\
\Delta_{P}= \\
200\end{array}$ & $85 \times 6.4$ & $\begin{array}{c}100 \times 50 \\
\text { Recta- } \\
\text { ngular }\end{array}$ & 20.0 & $\begin{array}{l}4.0-4.2 \\
(1) \\
3.3-3.4 \\
(\mathrm{~s})\end{array}$ & - \\
\hline \multirow[b]{2}{*}{9} & \multirow{2}{*}{$\begin{array}{c}\text { Tran-Nguyen } \\
\text { and Edil }(2011) \\
{[26]} \\
2 \text { zones }\end{array}$} & \multirow{2}{*}{$\begin{array}{c}h=530 \mathrm{~mm} \\
w_{e}=350 \mathrm{~mm} \\
t=130 \mathrm{~mm}\end{array}$} & $\begin{array}{c}\text { Reconstituted } \\
\text { HRK, } L_{L}=49 \%, \\
P_{L}=24 \%, G_{s}=2.59\end{array}$ & \multirow{2}{*}{$\begin{array}{c}\text { Sample with } w \text { equal } \\
\text { to the } \\
\text { field is placed on the } \\
\text { box, } \\
\text { placed in SZM } \\
\text { instrument coating, } \\
\text { and } \\
\text { compressed with } \\
\text { vibrator. }\end{array}$} & \multirow{2}{*}{$\begin{array}{c}P_{\mathrm{o}}= \\
25 \\
i= \\
20-30\end{array}$} & \multirow[b]{2}{*}{$100 \times 3.2$} & \multirow{2}{*}{$\begin{array}{c}15 \times 120 \\
\text { Recta- } \\
\text { ngular }\end{array}$} & \multirow[b]{2}{*}{$1.0-2.5$} & $\begin{array}{c}3.0 \\
\text { (HRK) }\end{array}$ & 1.03 \\
\hline & & & $\begin{array}{c}\text { Reconstituted } \\
\text { CID } L_{L}=49 \%, \\
P_{L}=20-25 \%, G_{s}= \\
2.71\end{array}$ & & & & & & $\begin{array}{c}4.2 \\
\text { (CID) }\end{array}$ & 1.25 \\
\hline
\end{tabular}




\begin{tabular}{|c|c|c|c|c|c|c|c|c|c|c|}
\hline \multirow[b]{2}{*}{ No. } & \multirow[b]{2}{*}{ Researchers } & \multirow[b]{2}{*}{$\begin{array}{c}\text { Tank } \\
\text { Dimension }\end{array}$} & \multirow[b]{2}{*}{$\begin{array}{l}\text { Basic Soil } \\
\text { Properties }\end{array}$} & \multirow{2}{*}{ Sample Preparation } & Stress & \multirow{2}{*}{$\begin{array}{c}\text { PVD } \\
\text { Dimension }\end{array}$} & \multirow{2}{*}{$\begin{array}{c}\text { Mandrel } \\
\left(\mathrm{mm}^{2}\right)\end{array}$} & \multirow{2}{*}{$\begin{array}{c}\text { Speed of } \\
\text { Installation }\end{array}$} & \multirow{2}{*}{$\begin{array}{c}\text { Extent } \\
\text { Ratio } \\
s^{\prime}=r_{s} / r_{m} \\
o r \\
s= \\
r_{s} / r_{w}^{*}\end{array}$} & \multirow[b]{2}{*}{$\begin{array}{c}\text { Permeability } \\
\text { Ratio } \\
\boldsymbol{\kappa}=\boldsymbol{k}_{h} / \boldsymbol{k}_{s}\end{array}$} \\
\hline & & & & & $(\mathrm{kPa})$ & & & & & \\
\hline 10 & $\begin{array}{l}\text { Ghandeharioon } \\
\text { et al. }(2012) \text { [27] } \\
3 \text { zones }\end{array}$ & $\begin{array}{c}h=900 \mathrm{~mm} \\
d=650 \mathrm{~mm} \\
h / d=1.38\end{array}$ & $\begin{array}{c}\text { Reconstituted } \\
\text { Lucustrine } \\
L_{L}=55 \%, P_{L}=27 \\
\%, e=1.46\end{array}$ & $\begin{array}{c}\text { Sample is mixed with } \\
\text { water until } w=1.1 \\
\mathrm{x} L_{L}, \text { placed in cell } \\
\text { consolidometer } \\
\text { layer by layer, and } \\
\text { compacted. }\end{array}$ & $\begin{array}{c}P_{o}= \\
20 \\
\Delta_{P}= \\
50\end{array}$ & $100 \times 4$ & - & - & 2.65 & $\begin{array}{c}\mathrm{k}_{\mathrm{h}} / \mathrm{k}_{\mathrm{v}}= \\
1.2-1.6\end{array}$ \\
\hline 11 & $\begin{array}{l}\text { Chai et al. } \\
\text { et al.(2013) [28] } \\
2 \text { zones }\end{array}$ & $\begin{array}{c}h=700 \mathrm{~mm} \\
d=450 \mathrm{~mm} \\
h / d=1.56\end{array}$ & $\begin{array}{c}\text { Reconstituted Soft } \\
\text { Bangkok Clay } \\
w=113 \%, \\
L_{L}=104 \%, P_{L}= \\
45 \%, \\
G_{s}=2.66, \gamma=14.7 \\
\mathrm{kN} / \mathrm{m}^{3} \\
\end{array}$ & $\begin{array}{c}\text { Samples are placed in } \\
\text { cell consolido- } \\
\text { meter layer by layer. }\end{array}$ & $\begin{array}{c}P_{o}= \\
50 \\
\Delta_{P}= \\
100\end{array}$ & $50 \times 3.5$ & $\begin{array}{c}81.9 \\
\times 18.2 \\
\text { Recta- } \\
\text { ngular }\end{array}$ & - & 2.0 & 3.0 \\
\hline 12 & $\begin{array}{l}\text { Rujikiatkamjorn } \\
\text { et al.(2014) [29] } \\
3 \text { zones }\end{array}$ & $\begin{array}{c}h=561 \mathrm{~mm} \\
d=345 \mathrm{~mm} \\
h / d=1.60\end{array}$ & $\begin{array}{c}\text { Undisturb Bulli } \\
\text { Clay } \\
\begin{array}{c}w=41 \%, L_{L}= \\
50 \%, P_{L}=25 \%, \\
G_{s}=2.62, \gamma=18.5 \\
\mathrm{kN} / \mathrm{m}^{3}\end{array}\end{array}$ & \begin{tabular}{|} 
The soil around the \\
sample is dug and \\
cut from base, \\
wrapped to \\
prevent loss of $w$, \\
stored in a humidity- \\
controlled room, and \\
placed into cell \\
consolidometer.
\end{tabular} & $\begin{array}{c}P_{o}= \\
20 \\
\Delta_{P}= \\
200\end{array}$ & $w_{e}=50$ & $\begin{array}{l}55 \times 5 \\
\text { Recta- } \\
\text { ngular }\end{array}$ & - & 3.7 & $1.33-2.85$ \\
\hline 13 & $\begin{array}{c}\text { Indraratna et al } \\
(2015)[30] \\
2 \text { zones }\end{array}$ & $\begin{array}{c}h=25,4 \mathrm{~mm} \\
d=63,5 \mathrm{~mm} \\
h / d=0.40\end{array}$ & 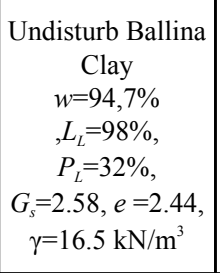 & $\begin{array}{c}\text { Samples for } \\
\text { Oedometer testing } \\
\text { are collected from } \\
\text { around the PVD } \\
\text { installed in the field. } \\
\text { A series } \\
\text { oedometer testing is } \\
\text { performed. }\end{array}$ & $\begin{array}{l}\Delta_{P}= \\
200\end{array}$ & $100 \times 3$ & $\begin{array}{c}120 \times 60 \\
\text { Recta- } \\
\text { ngular }\end{array}$ & - & 6.3 & 2.7 \\
\hline 14 & $\begin{array}{l}\text { Joseph et al. } \\
(2015)[31] \\
2 \text { zones }\end{array}$ & $\begin{array}{c}h=600 \mathrm{~mm} \\
d=600 \mathrm{~mm} \\
h / d=1.00\end{array}$ & $\begin{array}{c}\text { Reconstituted } \\
\text { Cochin Marine } \\
\text { Clay } \\
\mathrm{W}=112 \%, L_{L}= \\
156 \%, \\
P_{L}=34 \%, G_{s}= \\
2.62\end{array}$ & $\begin{array}{c}\text { Sample is placed into } \\
\text { the tank of } \\
\text { consolidometer, with } \\
w=L_{L} \text { layer by } \\
\text { layer. }\end{array}$ & $\begin{array}{c}P_{o}=5 \\
\Delta_{P}= \\
120\end{array}$ & $\begin{array}{c}\mathrm{SD} \\
\operatorname{diam} .=46 \\
\mathrm{~mm}\end{array}$ & $\begin{array}{l}\text { Circular } \\
\text { diam. }= \\
50 \mathrm{~mm}\end{array}$ & $\begin{array}{l}\text { hammer } \\
w_{\mathrm{i}}=2.6 \mathrm{~kg} \\
h_{i}=30 \mathrm{~cm}\end{array}$ & $\begin{array}{c}5.0- \\
6.0\end{array}$ & $1.3-1.4$ \\
\hline 15 & $\begin{array}{l}\text { Pajouh et al. } \\
(2015)[32] \\
2 \text { zones }\end{array}$ & $\begin{array}{c}h=200 \mathrm{~mm} \\
d=250 \mathrm{~mm} \\
h / d=0,80\end{array}$ & $\begin{array}{c}\text { Reconstituted } \\
\text { Kaolinite, } \\
\text { bentonite } \\
w=120 \%, L_{L}= \\
67-87 \% \\
P_{L}=27-34 \%, \\
P_{I}=40-43 \%\end{array}$ & $\begin{array}{c}\text { Samples are mixed } \\
\text { with water to } w=(1.4 \\
\text {-1.8) } L_{L} \text {, placed into } \\
\text { Rowe cell, } \\
\text { given stress cell }=110 \\
\mathrm{kPa} \text {,and back pressure } \\
=100 \mathrm{kPa} \text { for } \\
\text { saturation. }\end{array}$ & $\begin{array}{c}P_{o}= \\
20 \\
\Delta_{P}= \\
200\end{array}$ & $\begin{array}{c}\mathrm{SD} \\
\operatorname{diam} .=22 \\
\mathrm{~mm}\end{array}$ & $\begin{array}{c}\text { Circular } \\
\text { diam. }= \\
25 \mathrm{~mm}\end{array}$ & - & $3.0 *$ & 4.0 \\
\hline \multirow{2}{*}{16} & \multirow{2}{*}{$\begin{array}{l}\text { Sengul et al. } \\
(2016)[33] \\
2 \text { zones }\end{array}$} & \multirow{2}{*}{$\begin{array}{l}h=530 \mathrm{~mm} \\
w_{e}=350 \mathrm{~mm} \\
t=130 \mathrm{~mm}\end{array}$} & $\begin{array}{c}\text { Reconstituted } \\
\text { HRK, } L_{L}=51 \%, \\
P_{L}=26 \%, I_{P}=25 \%, \\
G_{s}=2.60 \\
\end{array}$ & \multirow{2}{*}{$\begin{array}{c}\text { Samples with } w \text { equal } \\
\text { to the } \\
\text { field are placed on the } \\
\text { box, } \\
\text { placed in SZM } \\
\text { instrument coating, } \\
\text { and compressed with } \\
\text { vibrator. }\end{array}$} & \multirow{2}{*}{$\begin{array}{c}P_{o}= \\
25 \\
\Delta_{h}=50\end{array}$} & \multirow{2}{*}{$130 \times 18$} & \multirow{2}{*}{$\begin{array}{l}120 \times 15 \\
\text { Recta- } \\
\text { ngular }\end{array}$} & \multirow{2}{*}{$2-5$} & 3.3 & 2.0 \\
\hline & & & $\begin{array}{c}\text { Reconstituted } \\
\text { CID, } \mathrm{L}_{\mathrm{L}}=51 \%, \\
P_{L}=30 \%, I_{P}=21 \%, \\
G_{s}=2.76\end{array}$ & & & & & & $\begin{array}{l}2.3- \\
2.4\end{array}$ & $2.86-3.13$ \\
\hline
\end{tabular}


(Table 2) contd.....

\begin{tabular}{|c|c|c|c|c|c|c|c|c|c|c|}
\hline \multirow[b]{2}{*}{ No. } & \multirow[b]{2}{*}{ Researchers } & \multirow[b]{2}{*}{$\begin{array}{c}\text { Tank } \\
\text { Dimension }\end{array}$} & \multirow[b]{2}{*}{$\begin{array}{l}\text { Basic Soil } \\
\text { Properties }\end{array}$} & \multirow{2}{*}{ Sample Preparation } & Stress & \multirow{2}{*}{$\begin{array}{c}\text { PVD } \\
\text { Dimension }\end{array}$} & \multirow[t]{2}{*}{ Mandrel } & \multirow{2}{*}{$\begin{array}{c}\text { Speed of } \\
\text { Installation }\end{array}$} & $\begin{array}{c}\text { Extent } \\
\text { Ratio }\end{array}$ & \multirow[b]{2}{*}{$\begin{array}{c}\text { Permeability } \\
\text { Ratio } \\
\qquad=k_{h} / k_{s}\end{array}$} \\
\hline & & & & & (kPa) & & & & $\begin{array}{c}s^{\prime}=r_{s} / r_{m} \\
o r \\
s= \\
r_{s} / r_{w} *\end{array}$ & \\
\hline 17 & $\begin{array}{l}\text { Choudhary et al. } \\
\text { (2016) [34] } \\
2 \text { zones }\end{array}$ & $\begin{array}{c}h=450 \mathrm{~mm} \\
d=650 \mathrm{~mm} \\
h / d=0.69\end{array}$ & $\begin{array}{c}\text { Reconstituted } \\
\text { Balina Clay } \\
w=94 \%, L_{L}= \\
98 \%, \\
P_{L}=32 \%, G_{s}=2.6\end{array}$ & $\begin{array}{c}\text { Clay is taken } 2 \mathrm{~m} \\
\text { below ground surface, } \\
\text { mixed with distilled } \\
\text { water with } w=1.4 L_{L} \text {, } \\
\text { placed into cell } \\
\text { consolidometer, and } \\
\text { given a light } \\
\text { vibration. }\end{array}$ & $\begin{array}{c}P o \\
=20 \\
\Delta_{P}= \\
60\end{array}$ & $100 \times 4$ & $\begin{array}{l}115 \times 10 \\
\text { Recta- } \\
\text { ngular }\end{array}$ & - & 2.5 & 1.3 \\
\hline
\end{tabular}

$h=$ height, $r=$ radius, $P_{o}=$ pra-consolidatiion stress, $(+\mathrm{v})=$ with PVD + vacuum preloading, $\Delta_{h}=$ hydraulic head,

$d,=$ diameter, $\mathrm{HRK}=$ Hydrite $\mathrm{R}$ Kaolinite, $\Delta_{P}=$ consolidation stress, $w_{i}=$ weight of hammer,

$w_{e},=$ width, $\mathrm{CID}=$ Craney Island Dredgings, $i=$ gradient hydraulic, $\mathrm{SD}=$ Sand Drain,

$t,=$ thickness, $\mathrm{SZM}=$ Smear Zone Model, $(l),(s)=$ mandrel long and short axis, $h_{i}=$ free fall height

The soil sample to be tested in the soil tank is divided into two types, i.e. disturbed and undisturbed. Because it is difficult and costly to make a sample that is not disturbed in a large scale, most laboratory tests use disturbed soil samples, employed among others by Sharma and Xiao (2000) [3], Chai et al., (2013) [28], and Sengul et al., (2016) [33]. On the other hand Rujikiatkamjorn et al., (2014) [29] used a relatively large of undisturbed sample with the aim of capturing more realistic smear zone characteristics within the intact soil structure, whereas Indraratna et al., (2015) [30] used a comparatively small, undisturbed sample directly from the field for oedometer testing. See Table 2 for more complete conditions and results of 17 previous laboratory studies on the smear zone.

The testing normally starts from the sample preparation stage. The sample preparation is carried out by mixing the soil with water until saturated, putting it into a consolidometer cell, extracting trapped air, and providing a preconsolidated compression. This stage is followed by the PVD or sand drained (SD) installation. The insertion of PVD (or SD) utilized a round or rectangular mandrel with a size smaller than the actual size. Also, the insertion speed in the laboratory is usually smaller than that in the field. Finally the consolidation stress representing the preloading is given. Based on the results of consolidation happening in the lab, the determination of the smear zone characteristics can be done. Columns 10-11 in Table 2 shows the results of the 17 previous laboratory on the characteristics of the smear zone composed mainly of soft soils.

\section{FIELD DATA vs LABORATORY RESULTS}

With reference to Tables 1 and 2, the extent ratio $s$ ' in the smear zone varies between 2.0-5.0 in the field using back calculation and 2.0-6.3 in the lab using laboratory apparatus. On the other side the permeability ratio $\kappa$ varies between 2.0-13.8 in the field using back calculation and 1.03-3.13 in the lab using laboratory apparatus.

It appears that the extent ratio $s$ ' resulting from both methods, field data back calculation and laboratory testing, yields comparable range. On the other hand the permeability ratio $\kappa$ resulting from the field data back calculation reveals a noticeably greater range if compared with that resulting from laboratory testing. The greater range of $\kappa$ values in the field indicates that the level of disturbance in the ground varies greatly between locations. Also, the discrepancy in $\kappa$ ranges implies that the laboratory testing can hardly model the high level disturbance of field conditions.

\section{DISCUSSION}

\subsection{Soil Conditions Before PVD Installation}

Soil samples are usually taken to the laboratory to determine the basic soil properties before PVD installation. See column 4 in Table 2 for the basic soil properties used in the lab.

Refering to the same soft soil prior to the PVD installation, Bo et al., (2001) [39] compared the values of permeability and consolidation coefficients ( $k$ and $c_{v}$ or $c_{h}$ respectively) from laboratory testings with those from field methods. They found that the corresponding values from laboratory results were noticeably lower than that from field testing. Similar results were previously reported by Balasubraniam et al., (1995) [40]. 
Chai and Miura (1999) [12] suggested that laboratory testing was the correct way to determine the value of $k_{s}$ for the smear zone, but the permeability coefficient value $k$ determined in laboratory before PVD installation would typically be lower than that in the field, resulting in smaller values of $\kappa$. Later Chu et al., (2002) [41] used Rowe consolidation cells in the laboratory to determine the coefficient consolidation values of $c_{h}$ and $c_{v}$; however, they concluded that the resulting $c_{h}$ and $c_{v}$ values could not represent the natural conditions of the soil. In short it should be pointed out that the important parameters of the soil including $c_{h}, c_{v}$, and $k$ determined in the laboratory can hardly represent the soil's natural conditions due to the inherently complex soil structures which cannot be represented within small specimen dimensions.

\subsection{Soil Conditions During and After PVD Installation}

\subsubsection{Land preparation for PVD Installation}

Land clearing and stripping is done in the field to clean the soil surface and remove layers of humus and other organic materials. A layer of sand blanket as thick as $0.5 \mathrm{~m}$ up to $1 \mathrm{~m}$ is then compacted on top of the soil surface. During the operation of PVD installation, the ground receives the moving load of the PVD rig; hence, the near top soil would be disrupted. This condition can hardly be modeled in the laboratory testing.

\subsubsection{Installation Depth of PVD}

Due to its very limited depth of less than $1 \mathrm{~m}$, soil in the laboratory receives a comparatively small amount of disturbance forces during PVD installation. On the other hand, Indraratna et al., (2015) [30] stated that the ground in the field gets distorted by the shear stress due to the PVD insertion. They also stated that the degree of distortion is more significant when the installed PVD gets longer. In this regard, laboratory testing is incapable of modelling the disturbing effects in the soil model caused by the depth of installation in the field. It should be noted that in the previous study sites, the depth of installation, indicated in column 3 in Table 1, varies between $8.0 \mathrm{~m}$ and 50.0 m dependent upon the field conditions, leading to the variation of the smear zone characteristics.

\subsubsection{Distance Between PVD}

Bo et al., (2003) [20] found that the extent ratio $s^{\prime}=4$ up to 7; then, for a PVD with an equivalent diameter of $d_{w}=$ $66 \mathrm{~mm}$, the smear extent $d_{s}=480 \mathrm{~mm}$ up to $528 \mathrm{~mm}$. Consequently PVDs installed with an interval of less than $1.0 \mathrm{~m}$ will start to have the interacting smear zones in which their disturbance effects on the permeability are unusually large, leading to a lower value of horizontal consolidation coefficient $c_{h}$. Balasubraniam et al. (1995) [40] and Chu et al., (2002) [41] reported the phenomenon of overlapping smear zones which cause the horizontal consolidation $c_{h}$ to be less than the vertical consolidation $c_{v}$, a condition at variance with the expectation. Walker and Indraratna (2007) [42] performed an analytical solution to assess the effect of overlapping smear zones and indicated that the critical, minimum effect radius was $0.55 r_{s}$ to $0.60 r_{s}$. The disturbance conditions due to the overlapping smear zones of the closely installed PVDs cannot be investigated in the laboratory since only single PVD has so far been modelled. At the previous study sites, the distance between PVDs, indicated in column 6 in Table 1, varies from $0.85 \mathrm{~m}$ to $3.0 \mathrm{~m}$ affecting the variation of the smear zone characteristics.

\subsubsection{Stiffness of Soil}

According to Sathananthan and Indraratna (2006) [23] and Sengul et al., (2016) [33] the extent ratio $s$ ' depends on soil stiffness. For more rigid soils, $s$ ' is greater than that for less rigid soils. With respect to the SPT values, the stiffness of the soil is not the same at every location and depth. Hence, the vertical variation in soil stiffness results in the variation of the smear extent in the vertical direction. Due the limited sample sizes, the soil stiffness variation is barely reflected in the laboratory.

\subsubsection{The dimension, Shape and Type of Mandrels}

The installation of PVD in the laboratory is generally performed using a mandrel that is reduced in size. In the field the commonly used sizes of mandrels are $120 \mathrm{~mm}$ x $60 \mathrm{~mm}$ for rectangular shape and $120 \mathrm{~mm}$ x $39 \mathrm{~mm}$ or $145 \mathrm{~mm} \times$ $90 \mathrm{~mm}$ for rhombic shape. The use of large mandrels serves to maintain the rig balance when installing PVD on thick soft soil layers. Sathananthan and Indraratna (2006) [23] affirmed that the extent ratio $s$ ' depends on the dimensions of the mandrel used. 
Bergado et al. (1991) [6] did experiments at a trial embankment by installing PVDs using large and small mandrels and indicated that the resulting dissipation of pore pressures were faster in locations using smaller mandrel. Their extensometer data showed slightly lower rates of settlement occurring in areas that use larger mandrels. Also, their back calculation at locations that use large mandrels yielded smaller horizontal consolidation coefficient $c_{h}$ values than those at locations using small mandrels.

It should be noted that trial embankment is costly but the smear zone parameters resulting from it better reflect the physical conditions of the field. Hence if fund is permitted, trial embankment is recommended to measure the settlement and excess porewater pressure to enable the back calculation method to search for the more accurate extent ratio $s$ ' and permeability ratio $\kappa$.

In relation with the shape effect, Bo et al., (2003) [20] explored that there are four shapes of mandrel namely rhombus, rectangle, square and circle and stated that the rhombus resulted in the least disturbance to the soil. In addition they indicated that the static rig used to drive PVD into the ground caused less interference if compared with the vibration rig. Likewise Tran-Nguyen and Edil (2011) [26] concluded that the size and the shape of mandrels were important factors affecting the value of $s^{\prime}$.

The dimension, shape and type of mandrel cannot be modelled precisely in the laboratory. At the previous study sites, the dimensions and shapes of the mandrels used in the field and in the lab vary as indicated in column 7 in Table $\mathbf{1}$ and in column 8 in Table 2 , consecutively.

\subsubsection{The Speed of PVD Installations in the Field}

The installation of PVD in the laboratory is normally performed at low speeds, ranging from 0.5 to $20 \mathrm{~mm} / \mathrm{s}$. Sathananthan and Indraratna (2006) [23] stated that the extent ratio $s$ depended on the speed of the PVD insertion. In contrast Rixner et al. (1986) [5] stated that PVD was installed in the field at a speed of 150 up to $600 \mathrm{~mm} / \mathrm{s}$. Hence the ground disturbance in the field is greater than that in the laboratory. In Tables $\mathbf{1}$ and $\mathbf{2}$ the speeds of PVD installation are given in column 10 for field and in column 9 for laboratory, respectively.

\subsubsection{Use of Reconstituted Soil Samples}

Liu and Carter (2000) [43] discussed how the behavior of soil structures of the original soils was different from that of the reconstituted soils. Tran-Nguyen and Edil (2011) [26] who used reconstituted samples revealed that the extent and permeability ratios measured in their laboratory results were at the lower limit reported in the literature. These may occur because the soils were very disturbed and had no structure, thus less susceptible to disturbance.

The laboratory testing using undisturbed soil samples was done by, among others, Rujikiatkamjorn et al., (2014) [29] who found that permeability reductions were almost twice as much as those using disturbed soil. Bo et al., (2003) [20] suggested that the smear zone could become larger in undisturbed soils due to the destruction of the soil structure. In any case the condition of the soil in the field is normally not disturbed, leading especially to the higher values of the permeability ratio $\kappa$.

\subsubsection{Total Height of Embankment}

Total height of embankment is directly responsible for the total working consolidation stress in the soil. Sharma and Xiao (2000) [3] and Sathananthan and Indraratna (2006) [23] confirmed that the permeability ratio $\kappa$ would decrease with the increasing pressure of consolidation. Most recently Sengul et al., (2016) [33] reassured the decrease of $k_{h} / k_{s}$ and $k_{h} / k_{t}$ in smear and transition zones with the increased consolidation stress. The variation of the total height of embankment in the previous study sites is given in column 9 in Table 1, ranging from $1.5 \mathrm{~m}$ up to $8.0 \mathrm{~m}$.

In the laboratory testing the total height of embankment is applied using the consolidation stress as indicated in column 6 in Table 2. Referring to the table, the consolidation stress ranges from $47.8 \mathrm{kPa}$ to $1102 \mathrm{kPa}$ indicating quite a large variation in terms of the total embankment heights which are modelled in the lab.

\subsubsection{The Ranges and Classification of 's' and $\boldsymbol{k}$ Values}

As mentioned earlier, the extent ratio $s$ ' values vary between $2.0-5.0$ and between $2.0-6.3$ based on field data back calculation and laboratory testing, consecutively. On the other hand, the $\kappa$ values vary between $2.0-13.8$ and 1.03 -3.13 based on field data back calculation and laboratory testing, consecutively. It can be seen that the ranges of $s$, values from both methods are relatively close since the key factor, the soil stiffness, is not disturbed much in both cases. 
In contrast, the range of $\kappa$ values from field data is significantly larger than that from laboratory testing due to the complexity and high level of disturbance in the field.

The range of the $s^{\prime}$ values can be classified as follows: $1<s^{\prime}<2$ low, $2 \leq s^{\prime}<4$ moderate, and $4 \leq s^{\prime}<7$ high. It is found that the values of $s^{\prime}$ based on field data back calculation and laboratory test results are mostly in the moderate range.

The range of $\kappa$ values can be classified as follow: $1<\kappa<4$ low, $4 \leq \kappa<7$ moderate, $7 \leq \kappa<10$ high, and $10 \leq \kappa<14$ very high. It is found that the values of $\kappa$ based on field data back calculation are mostly in the high to very high ranges, while the $\kappa$ values from laboratory test results are mostly in the low range.

\section{A CASE STUDY}

The position of the case study is at the location of the multi purpose container yard in Kuala Tanjung Port which is is located on the east coast of North Sumatra, Indonesia, fronting the Strait of Malacca. Fig. (2) shows the 13.5 ha of the embankment area of the case study site taken during preloading in June 2017.

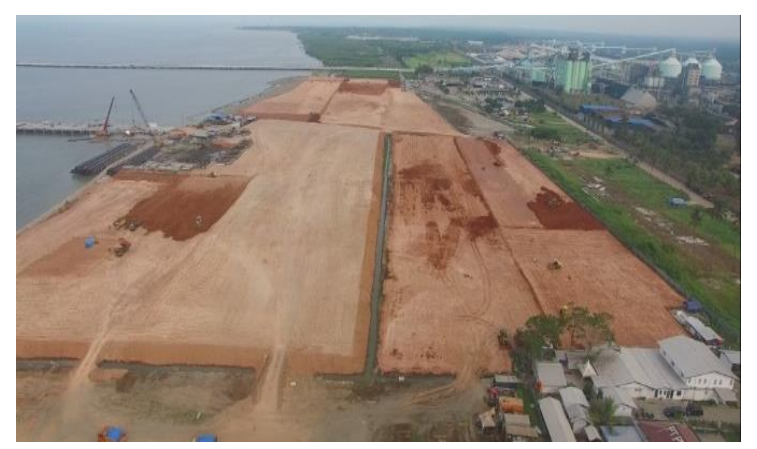

Fig. (2). Photograph of the embankment area of the case study.

The soil investigation was carried out using a machine drill, SPT, undisturbed and disturb samplings, DCP, and laboratory testing. From the results of the soil investigation it is known that the basic soil layer is composed of soft clay and very soft clay as thick as $10 \mathrm{~m}$, with $\mathrm{SPT} \leq 2$. This clay layer sits above a sandy clay layer having a medium consistency of $7 \mathrm{~m}$ thick with $4 \leq \mathrm{SPT} \leq 5$. This sandy clay layer is located above a dense sand layer of $3 \mathrm{~m}$ thick with $10 \leq \mathrm{SPT} \leq 30$, below which a very dense sand layer of $10 \mathrm{~m}$ thick rests with $\mathrm{SPT} \geq 60$.

A geotextile separator is used over the 13.5 ha of the embankment area to separate the base ground with the embankment material. The data related to the PVD work in the case study site can be seen in the last row in Table $\mathbf{1}$. The geotechnical instruments used for the analysis encompassed 7 piezometers, 7 water standpipes, 44 settlement plates, and 8 inclinometers. The total height of the embankment, which has an average of $5.2 \mathrm{~m}$, was achieved gradually in 14 stages as indicated in Fig. (3).

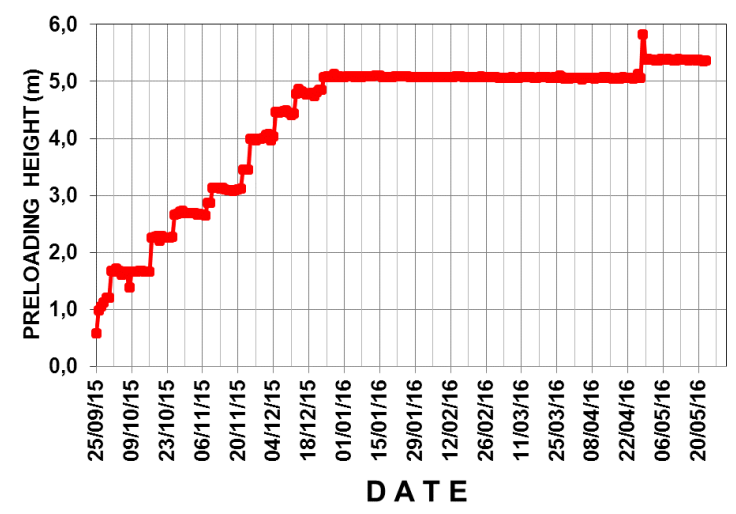

Fig. (3). Construction stages at the trial embankment location.

The consolidation induced settlement is calculated using the 1-D consolidation with the load distribution following the $2 \mathrm{~V}: 1 \mathrm{H}$ ratio and $c_{h}=1,35 c_{v}$. The degree of consolidation of the embankment is estimated by taking the smear zone 
effect into account and ignoring the well resistance.

The characteristics of the smear zone are determined through the back-calculation method using time dependent settlement data obtained from the case study site (Fig. 4). For the case of constant permeability in the smear zone, the settlement data are fitted with Eq. (1) using the parameter $m$ of Eq. (4). For the case of parabolic permeability in the smear zone, they are fitted with Eq. (1) using the parameter $m$ given by Eq. (5). Lastly, for linier permeability in the smear zone, they are fitted with Eq. (1) using the paramater $m$ given by Eq. (6).

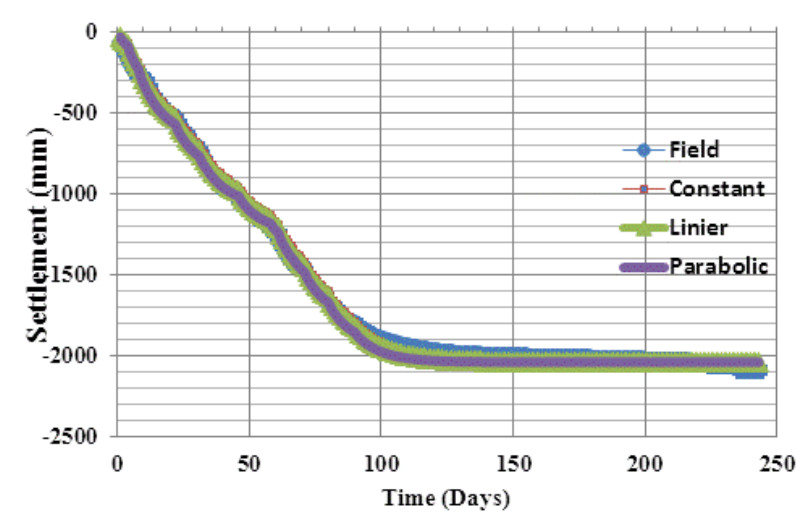

Fig. (4). Time-settlement relationships of field data and back calculation results.

Plotted against the field settlement data in Fig. (4), the results of the consolidation for the three permeabilities are obtained using the value of $s^{\prime}=3$ and $\kappa=7$. If the results in the figure are checked in detail, it can be seen that the discrepancies appear to be significant on days 85 up 135. However, the largest discrepancy is found to be with the parabolic permeability, followed by the linear and the constant permeabilities in a decreasing order. It is noteworthy that the extent ratio $s^{\prime}=d_{s} / d_{m}=3$ is the value suggested by Chai and Miura (1999) [12] for locations with no test data. Meanwhile the value for permeability ratio $\kappa=\left(k_{h} / k_{s}\right)=7$ can be considered as a high representative value that reflects the level of disturbance in the soil during PVD installation.

\section{CONCLUSION}

In this study we have documented and evaluated the values of smear zone parameters, namely the extent ratio s' and the permeability ratio $\kappa$, resulting from field investigation and laboratory testing. The assessment on the causes of the variation of the values of the smear zone parameters is made particularly with relation to the different conditions in the field and in the laboratory. A case study is given here to illustrate the procedure to estimate the values of both parameters by field data back calculation. The concluding findings can be summarized as follows.

Smear zone parameter values are quite different in accordance with their locations. With reference to the results of the previous studies it is found that the extent ratio $s$ ' values vary between $2.0-5.0$ and between 2.0 - 6.3 based on the back calculation and laborary testing, consecutively. On the other hand, the permeability ratio $\kappa$ values based on the field data back calculation range from 2.0 to 13.8 which are quite large if compared with the $\kappa$ range from 1.03 to 3.13 based on laboratory testing.

Three causes of the $s^{\prime}$ and $\kappa$ variations are suggested here. First, the laboratory testing is unable to model the complexity of field conditions. Second, there are no standards established to be used as references in the laborary testing. Third, the level of disturbance in the field is much greater than that in the laboratory testing and differs at each research location.

If fund is permitted, it is recommended that a trial embankment be applied using the same methods, materials and equipment that will be used on the actual embankment. Then smear zone parameter values will likely be obtained with a better accuracy for the real application of PVD.

\section{CONSENT FOR PUBLICATION}

Not applicable. 


\section{CONFLICTS OF INTEREST}

The authors declare no conflict of interest, financial or otherwise.

\section{ACKNOWLEDGEMENTS}

The authors gratefuly acknowledge the financial assistance from the University of Sumatera Utara (Research Contracts Fiscal Year 2018, Number: 2590/UN5.1.R/PPM/2017 dated March 16, 2018). Appreciation is given to PT. Prima Multi Terminal which provided the authors with data for the case study. The contribution from Nurhayani Simamora in typing and formating the paper is also appreciated.

\section{REFERENCES}

[1] R.D. Holtz, M.B. Jamiolkowski, R. Lancellotta, and R. Pedroni, "Prefabricated vertical drains: Design and performance," Oxford, UK: Butterworth-Heinemann, 1991.

[2] B. Indraratna, C. Rujikiatkamjorn, and I. Sathananthan, "Radial consolidation of clay using compressibility indices and varying horizontal permeability", Can. Geotech. J., vol. 42, pp. 1330-1341, 2005. [http://dx.doi.org/10.1139/t05-052]

[3] J.S. Sharma, and D. Xiao, "Characterization of a smear zone around vertical drains by large-scale laboratory tests", Can. Geotech. J., vol. 37, no. 6, pp. 1265-1271, 2000. [http://dx.doi.org/10.1139/t00-050]

[4] D. Basu, and M. Prezzi, "Effect of the smear and transition zones around prefabricated vertical drains installed in triangular pattern on the rate of soil consolidation", ASCE. J Geomech., vol. 7, no. 1, pp. 34-43, 2007. [http://dx.doi.org/10.1061/(ASCE)1532-3641(2007)7:1(34)]

[5] J.J. Rixner, S.R. Kramer, and A.D. Smith, "Prefabricated vertical drains", Technical Report, vol. II: Summary research efforts, Federal Highway Administration Report FHWA/RD-86/169, 1986.

[6] D.T. Bergado, H. Asakami, M.C. Alfaro, and A.S. Balasubramaniam, "Smear effects of vertical drains on soft Bangkok clay", Geotech. Eng. $A S C E$, vol. 117, no. 10, pp. 1509-1530, 1991.

[7] D.T. Bergado, A.S. Enriquez, C.L. Sampaco, M.C. Alfaro, and A.S. Balasubramaniam, "Inverse analysis of geotechnical parameters on improved soft Bangkok clay", J. Geotech. Eng., vol. 118, pp. 1012-1030, 1992. [http://dx.doi.org/10.1061/(ASCE)0733-9410(1992)118:7(1012)]

[8] D.T. Bergado, and M.A.B. Patawaran, "Recent developments of ground improvement with PVD on soft Bangkok clay", in Proc. Intl. Seminar on Geotechnics in Kochi, Japan, 2000.

[9] J. Saowapakpiboon, D.T. Bergado, S. Youwai, J.C. Chai, P. Wanthong, and P. Voottipruex, "Measured and predicted performance of prefabricated vertical drains (PVDs) with and without vacuum preloading", Geotext. Geomembr., vol. 28, pp. 1-11, 2010. [http://dx.doi.org/10.1016/j.geotexmem.2009.08.002]

[10] H. Hiep, and S.G. Chung, "Back-analysis of geotechnical parameters on PVD-improved ground in the Mekong Delta", Geotext. Geomembr., vol. 46, pp. 402-413, 2018. [http://dx.doi.org/10.1016/j.geotexmem.2018.03.005]

[11] B. Indraratna, and I.W. Redana, "Numerical modelling of vertical drains with smear zone and well resistance installed in soft clay", Can. Geotech. J., vol. 37, no. 1, pp. 132-145, 2000. [http://dx.doi.org/10.1139/t99-115]

[12] J.C. Chai, and N. Miura, "Investigation of factors affecting vertical drain behavior", J. Geotech. Geoenviron. Eng. ASCE, vol. 125, no. 3, pp. 216-226, 1999.

[13] J.C. Chai, N. Miura, S. Sakajo, and D.T. Bergado, "Behavior of vertical drain improved subsoil under embankment loading", Soil Found., vol. 35, pp. 49-61, 1995.

[http://dx.doi.org/10.3208/sandf.35.4_49]

[14] J.C. Chai, D.T. Bergado, N. Miura, and S. Sakajo, "Black calculated field effect of vertical drain", in Second International Conference on Soft Soil Engineering, Nanjing, China, pp. 270-275, 1996.

[15] J.C. Chai, S.L. Shen, N. Miura, and D.T. Bergado, "Simple method of modeling PVD improved subsoil", J. Geotech. Geoenviron. Eng., vol. 127, no. 11, pp. 965-972, 2001.

[http://dx.doi.org/10.1061/(ASCE)1090-0241(2001)127:11(965)]

[16] B. Indraratna, A. Aljorany, and C. Rujikiatkamjorn, "Analytical and numerical modelling of consolidation by sand drains beneath a circular embankment", Int. J. Geomech., vol. 8, no. 3, pp. 199-206, 2008. [http://dx.doi.org/10.1061/(ASCE)1532-3641(2008)8:3(199)] 
[17] A.P. Pajouh, B. Fatahi, and H. Khabbaz, "Numerical analysis to quantify the Influence of smear zone characteristics on preloading design in soft clay", In: Proceedings of the $18^{\text {th }}$ International Conference on Soil Mechanics and Geotechnical Engineering, Paris, France, 2013, pp. 2573-2576.

[18] A.P. Pajouh, B. Fatahi, P. Vincent, and H. Khabbaz, "Analyzing consolidation data to predict smear zone characteristics induced by vertical drain installation for soft soil improvement", Geomech. Eng., vol. 7, pp. 105-131, 2014. [http://dx.doi.org/10.12989/gae.2014.7.1.105]

[19] A.P. Pajouh, B. Fatahi, P. Vincent, and H. Khabbaz, "Trial embankment analysis to predict smear zone characteristics induced by prefabricated vertical drain Installation", In: Geotech Geol Eng, Springer International Publishing Switzerland, 2014.

[20] M.W. Bo, J. Chu, and V. Choa, "Soil improvement: Prefabricated vertical drain techniques,” Thompson, Singapore, pp. 341 , 2003.

[21] B. Indraratna, and I.W. Redana, "Laboratory determination of smear zone due to vertical drain installation,", J. Geotech. Geoenviron. Eng., vol. 124 , no. 2 , pp. 180-184, 1998. [http://dx.doi.org/10.1061/(ASCE)1090-0241(1998)124:2(180)]

[22] B. Indraratna, and C. Rujikiatkamjorn, "Laboratory determination of efficiency of prefabricated vertical drains incorporating vacuum preloading", in $15^{\text {th }}$ Southeast Asian geotechnical conference, vol. 1, no. 2, pp. 453-456, 2004.

[23] I. Sathananthan, and B. Indraratna, "Laboratory evaluation of smear zone and correlation correlation between permeability and moisture content", J. Geotech. Geoenviron. Eng. ASCE, vol. 132, no. 7, pp. 942-945, 2006.

[24] Z. Fang, and J.H. Yin, "Physical modelling of consolidation of Hong Kong marine clay with prefabricated vertical drains", Can. Geotech. J., vol. 43, pp. 638-652, 2006. [http://dx.doi.org/10.1139/t06-021]

[25] D.H. Shin, C. Lee, J.S. Lee, and W. Lee, "Detection of smear zone using micro-cone and electrical resistance probe", Can. Geotech. J., vol. 46, pp. 719-726, 2009. [http://dx.doi.org/10.1139/T09-020]

[26] H.H. Tran-Nguyen, and T.B. Edil, "The characteristic of PVD smear zone", Geo-Frontier 2011 ASCE, pp. 748-757, 2011.

[27] A. Ghandeharioon, B. Indraratna, and C. Rujikiatkamjorn, "Laboratory and finite-element investigation of soil disturbance associated with the installation of mandrel-driven prefabricated vertical drains", J. Geotech. Geoenviron. Eng., vol. 138, no. 3, pp. 295-308, 2012. [http://dx.doi.org/10.1061/(ASCE)GT.1943-5606.0000591]

[28] J.C. Chai, D.T. Bergado, and S.L. Shen, "Modelling prefabricated vertical drain improved ground in plane strain analysis", Proc.- Inst. Civ. Eng., vol. 166, pp. 65-77, 2013.

[29] C. Rujikiatkamjorn, B. Indraratna, and M.D.W. Ardana, " Smear zone characterization associated with vertical drain installation", In: Proceedings of Soft Soils, 2014, pp. F1-1-F1-8.

[30] B. Indraratna, D. Perara, C. Rujikiatkamjorn, and R. Kelly, "Soil distubance analysis due to vertical drain instalation", in Proceedings of the institution of Civil Engineering, Geotech. Eng., vol. 168, no. 3, pp. 236-246, 2015. [http://dx.doi.org/10.1680/geng.14.00052]

[31] A. Joseph, S. Chandrakaran, N. Sanka, and B.T. Jose, "Laboratory evaluation of extent of smear zone due to columnar intrusion for Cochin marine clays", in $50^{\text {th }}$ Indian Geotechnical Conference, Pune, India, 2015.

[32] A.P. Pajouh, B. Fatahi, and H. Khabbaz, "Experimental and numerical investigations to evaluate two-dimensional modelling of vertical drain-assisted preloading", Int. J. Geomech., pp. 1-14, 2015.

[33] T. Sengul, T. Edil, and K. Ozaydin, "Laboratory determination of smear and transition zones due to prefabricated vertical drain installation", Mar. Georesour. Geotechnol., vol. 35, pp. 895-904, 2016. [http://dx.doi.org/10.1080/1064119X.2016.1256924]

[34] K. Choudhary, B. Indraratna, and C. Rujikiatkamjorn, "Pore pressure based method to quantify smear around a vertical drain", Géotechnique Letters, pp. 211-215, 2016.

[35] S. Hansbo, "Consolidation of fine-grained soils by prefabricated drains", in Proceedings $10^{\text {th }}$ International Conference on Soil Mechanics and Foundation Engineering, vol. 3, Stockholm, pp. 667-682, 1981.

[36] R. Walker, and B. Indraratna, "Vertical drain Consolidation with parabolic distribution of permeability in smear zone", J. Geotech. Geoenviron. Eng., vol. 132, no. 7, pp. 937-941, 2006. [http://dx.doi.org/10.1061/(ASCE)1090-0241(2006)132:7(937)]

[37] C. Rujikiatkamjorn, and B. Indraratna, "Design procedure for vertical drains considering a linear variation of lateral permeability within the smear zone", Can. Geotech. J., vol. 46, no. 3, pp. 270-280, 2009. [http://dx.doi.org/10.1139/T08-124]

[38] F. Tavenas, M. Tremblay, G. Larouche, and S. Leroieil, "In situ measurement of permeability in soft soil", In: ASCE Special Conference on Use of In-Situ Test in Geotechnical Engineering, 1986, pp. 1034-1048.

[39] M. W. Bo, J. Chu, and V. Choa, "Comparison of consolidation parameters measured by laboratory and in-situ tests," Can. Geotech. J., pp.1871-1874, 2001.

[40] A. S. Balasubramanian, D. T. Bergado, P. V. Long, and Thayalan, "Experiences with sand drains and prefabricated vertical drains in ground 
improvement of soft clays", In: Seminar on Eng.for Coastal Development, Singapore, 1995, pp. s29-s40.

[41] J. Chu, M.W. Bo, M.F. Chang, and V. Choa, "The consolidation and permeability properties of Singapore marine clay", J. Geotech. Geoenviron. Eng., vol. 128, no. 9, pp. 724-732, 2002.

[http://dx.doi.org/10.1061/(ASCE)1090-0241(2002)128:9(724)]

[42] R. Walker, and B. Indraratna, "Vertical drain consolidation with overlapping smear zones", Geotechnique, vol. 57, pp. 463-467, 2007. [http://dx.doi.org/10.1680/geot.2007.57.5.463]

[43] M.D. Liu, and J.P. Carter, "Modelling the destructuring of soils during virgin compression", Geotechnique, vol. 50, no. 4, pp. 479-483, 2000. [http://dx.doi.org/10.1680/geot.2000.50.4.479]

\section{(C) 2018 Iskandar et al.}

This is an open access article distributed under the terms of the Creative Commons Attribution 4.0 International Public License (CC-BY 4.0), a copy of which is available at: (https://creativecommons.org/licenses/by/4.0/legalcode). This license permits unrestricted use, distribution, and reproduction in any medium, provided the original author and source are credited. 